УДК 331.102 .1

\title{
Д.М. Маликова
}

\section{РАЗРАБОТКА МЕТОДОЛОГИИ ОЦЕНКИ ТЕХНОЛОГИЧНОСТИ ПРОМЫШЛЕННЫХ ИЗДЕЛИЙ В УСЛОВИЯХ ОПЫТНО-СЕРИЙНОГО ПРОИЗВОДСТВА}

Статья посвящена повышению достоверности комплексной количественной оценки показателей технологичности конструкции изделия в условиях опытно-серийного производства с учетом конкретных производственнотехнологических условий предприятия путем решения задачи объективного выбора показателей технологичности. Для достижения поставленной цели были решены следующие задачи: исследованы показатели технологичности конструкций изделий, их количественная оценка; разработана математическая модель формирования конструктивных показателей технологичности, осуществлен выбор оптимального набора показателей технологичности для конкретного изделия; разработана методика количественной оценки и экспертного выбора показателей технологичности по их весам; разработан алгоритм определения интегрального показателя технологичности изделия; применен разработанный алгоритм интегральной оценки показателей технологичности изделия. Практическая значимость работы заключается в апробации авторской методики экспертного выбора и определения интегрального показателя технологичности. Рассматриваемый показатель является одним из элементов повышения эффективности деятельности предприятия, особенно в условиях опытно-серийного производства. Разработанная автором методика позволяет более достоверно оценивать технологичность изделия на начальных этапах проектирования. Положительный результат получен путем экспертного отбора показателей, учитывающих особенности конструкции и технологического уровня изготовителя. Кроме того, предложенная методика дала возможность выявить резервы повышения технологичности конструкции изделия. Применение авторской методики способствует сокращению сроков и затрат, связанных с освоением и производством нового изделия.

Ключевые слова: опытно-серийное производство, технологичность изделия, экспертная оценка, ранжирование показателей, повышение технологичности, экономическая эффективность, финансовый результат, процесс труда.

DOI: $10.35634 / 2412-9593-2021-31-4-597-602$

Президент Российской Федерации Владимир Путин еще в 2016 г. поручил увеличить до 50 \% долю гражданской продукции предприятиям оборонно-промышленного комплекса. Однако, при решении вопроса перехода к выпуску высокотехнологичных изделий гражданского направления предприятия ОПК столкнулись с необходимостью сокращения временных и финансовых затрат на разработку и опытно-серийное производство изделий высокого качества.

В Советском Союзе разработка конструктивно новых изделий проводилась централизованно на всех уровнях: государственном, отраслевом, на предприятиях. Были разработаны специальные комплексы стандартов как для предприятий, выпускающих готовую продукцию, так и для научноисследовательских институтов. Этот комплекс стандартов представлял собой единую систему технологической подготовки производства, в которой формировались основные задачи обеспечения технологичности конструкции, определялась последовательность решения, предлагалась система количественных показателей, утверждались общие правила выбора на всех стадиях разработки конструкции. На данный момент все перечисленные выше вопросы стоят непосредственно перед предприятиями и организациями. Никто не готов передавать результаты интеллектуальной собственности другим предприятиям, являющимся также конкурентами на рынке. Производственные предприятия вынуждены самостоятельно выстраивать системы управления, начиная от конструирования новых изделий и организации опытно-серийного производства до послегарантийного обслуживания и утилизации изделий. При этом процесс труда на каждом из этапов жизненного цикла продукта должен быть организован с максимальной экономической эффективностью. Обобщенным критерием эффективности конкретного производства будет его рентабельность и положительный финансовый результат (чистая прибыль) предприятия.

Но оценка эффективности должна производиться еще до запуска технологических цепочек производства продукта. Основой успешного производства продукта может стать предварительная оценка уровня технологичности изделий. Эффективным способом определения степени технологичности разрабатываемой конструкции является сравнение с несколькими аналогами или вариантами конструкции. Оценить новое изделие одного типа и назначения можно различными методами количественной и качественной оценок технологичности изделия. Вопросы использования различных 
схем для выбора оптимальных показателей технологичности для конкретных изделий и конкретных организационно-технических условий их производства требуют выработки новых подходов, особенно для наукоемких отраслей промышленности.

Учитывая опыт отечественных предприятий можно сделать вывод, что отработка изделий на технологичность должна носить системный характер и проводиться по заранее разработанным алгоритмам. Предлагаемая методика должна иметь возможность подстраиваться к производственным условиям каждого конкретного предприятия. Требования единой системы технологической подготовки производства в принципе актуальны в условиях современного производства. При этом предполагается решение нескольких задач [1]:

- отработка на технологичность на всех этапах жизненного цикла изделия;

- выявление всех показателей технологичности;

- количественная оценка показателей технологичности;

- обеспечение технологичности различных промышленных изделий;

- разработка методических рекомендаций для конструкторов и технологов по всем сопутствующим вопросам.

Для обеспечения возможности нормирования показателей предложена система оценок технологичности конструкции изделий в процессе разработки [2]. Под оценкой технологичности конструкции понимают ряд мероприятий, связанных между собой. Они включают алгоритм определения технологичности составных частей изделия путем сопоставления наиболее важных параметров разрабатываемого изделия и аналога. Полученные результаты подвергаются глубокому анализу специалистами в данной области. Далее информация представляется в оптимальной форме для принятия управленческих решений. По результатам анализа проектируемого изделия принимается решение о возможном совершенствовании технологии изготовления и конструкции в целом [3].

Эффективность отработки на технологичность зависит от быстрой и адекватной оценки конструкции. Непосредственно процесс оценки технологичности представляет собой комплексную систему и занимает важное место на всех этапах производства.

При определении технологичности конструкции изделия путем качественной и количественной оценки показателей появляется возможность предсказать наиболее важные свойства перспективных изделий с последующим их сравнением со свойствами изделий аналогов и сформировать интегральный показатель технологичности.

Нормативные и технические документы не регламентируют процедуры определения и отбора показателей технологичности конструкции изделий. Имеющиеся рекомендации предлагают частные или относительные показатели технологичности с указанием их «веса» в виде коэффициентов. Количество самих показателей не превышает 7 в зависимости от конкретного изделия [4].

Существующие методики по выбору показателей технологичности конструкции изделий имеют целый ряд недоработок:

- необходимо очень чёткое отнесение конструкции изделия к строго определенному классу (так как номенклатура изделий имеет большое разнообразие, а их функциональное назначение определить чаще всего невозможно, то можно сделать вывод о том, что границы классов сегодня «размыты»);

- «веса» показателей определены без учёта стадии разработки изделия.

Для того чтобы решить задачу количественного определения технологичности конструкции изделия была разработана авторская методика экспертных оценок. Она позволяет проводить оценку технологичности конструкции более гибко, при этом широко использовать как выбор состава показателей технологичности, так и определенные коэффициенты «веса» [5].

Предлагаемая методика состоит из нескольких этапов. Первый этап заключается в разработке системы определенных показателей технологичности конструкции изделия, особенно в условиях опытно-серийного производства. От адекватности и важности показателей, входящих в систему на стадиях опытно-серийного производства изделия, зависит надежность оценок.

Далее эксперт оценивает значимость каждого критерия из списка показателей и удаляет «ненужные», малозначимые для оцениваемого данного конкретного изделия. Эксперт вносит дополнительные показатели, аргументируя свои действия в письменной форме, если в представленной системе не отражены наиболее важные показатели технологичности конструкции. Рабочая группа собирает данные от экспертов по показателям технологичности конструкции и систематизирует их. После анализа всех показателей готовится окончательная классификация показателей технологичности конструкции, которая будет использоваться для последующего формирования конкретных коэффициентов и определения их 
Разработка методологии оценки технологичности промышленных изделий...

значимости. Наиболее опытным экспертам предлагается сформировать матрицу «парных сравнений». Матрица заполняется по заголовкам столбцов и строк из показателей технологичности конструкции [6].

Если эксперты внутри рабочей группы не могут прийти к согласию, то необходимо провести второй этап экспертизы. Перед его проведением рабочая группа должна выявить «неадекватных» экспертов, формирующих «диаметрально противоположные» точки зрения, и усреднить мнение экспертов. Перед вторым этапом экспертам доводится усредненная оценка, обоснованная специалистами. Обоснования проводятся анонимно, без указания конкретных экспертов. Далее эксперты корректируют данные оценки.

Полученные экспертные мнения представляют в виде гистограммы показателей технологичности конструкции. На гистограмме значимые показатели технологичности имеют значения больше среднего уровня. Величина интегрального показателя технологичности конструкции сравнивается с показателем из технического задания на разработку изделия. Предложенная конструкция считается технологичной, если полученное расчётное значение выше базового [6].

Исследование предлагаемой методики проводилось на ООО «Русимпорт» (г. Ижевск). Целью экспериментального исследования была проверка работоспособности модели оценки ТК и отработка методики выбора оптимальных показателей технологичности и получения интегральной оценки ТК изделия электронный логистический инструментальный навигатор ЭЛИН-1 в 5 вариантах изготовления, тем самым подтвердить эффективность авторской методики.

Предусматривалось решение нескольких задач:

- формирование экспертной и рабочей групп;

- разработка алгоритма определения показателей технологичности конструкции;

- статистическая обработка результатов экспертной оценки;

- количественная оценка интегрального показателя технологичности изделия;

- сравнение значений показателей технологичности конструкции;

- принятие решения об изменении конструкции для повышения её технологичности.

На предприятии была сформирована группа экспертов из следующих специалистов:

- двух инженеров-конструкторов;

- двух инженеров-технологов;

- одного инженера по нормированию труда.

Для ознакомления было представлено техническое задание с техническими характеристиками разрабатываемого изделия и комплект конструкторской документации. Экспертам было сделано предложение выбрать из 32 различных показателей технологичности конструкции. Показатель технологичности конструкции включался в перечень, сформированный на предварительном этапе, если его рекомендовали большинство экспертов. Эксперты на основе полученной классификации показателей и значимость показателей выбрали 19 показателей технологичности конструкции. Некоторые показатели [8] были отвергнуты экспертами, например:

1) Коэффициент повторяемости;

2) Коэффициент применяемости элементов;

3) Коэффициент применяемости деталей миниатюризации;

4) Коэффициент повторяемости материалов;

5) Коэффициент повторяемости отверстий;

6) Коэффициент использования материалов;

7) Стоимость элементной базы собственного изготовления;

8) Коэффициент сложности обработки;

9) Коэффициент автоматической установки элементов;

10) Коэффициент согласованности технологического оснащения;

11) Коэффициент применения групповых методов пайки;

12) Коэффициент автоматизированной подготовки элементов к монтажу и сборке;

13) Коэффициент автоматизации технологических процессов;

14) Коэффициент автоматизации технологических процессов контроля;

15) Коэффициент параллельности сборки;

16) Коэффициент автоматизации контроля и настройки.

На втором этапе выбора показателей ТК экспертам был предложен список из 19 позиций. Эксперты ранжировали показатели методом «парных сравнений», исходя из их информативности (табл. 1). 
Таблица 1

Результаты распределения показателей технологичности изделия ЭЛИН-1

\begin{tabular}{|c|c|c|c|c|c|c|c|c|}
\hline \multirow{2}{*}{$\begin{array}{l}\text { № } \\
\Pi / \Pi\end{array}$} & \multirow{2}{*}{ Наименование показателя } & \multicolumn{5}{|c|}{$\begin{array}{c}\text { Число предпочтений } \\
\text { показателя экспертом } p_{\mathrm{ik}}\end{array}$} & \multirow{2}{*}{$\begin{array}{c}\text { Суммарное } \\
\text { число } \\
\text { вредпочтений } \\
\mathrm{S}_{\mathrm{i}}\end{array}$} & \multirow{2}{*}{$\begin{array}{c}\text { Среднее } \\
\text { число пред- } \\
\text { почтений } \\
\mathrm{S}_{\text {iсред. }} \\
\end{array}$} \\
\hline & & 1 & 2 & 3 & 4 & 5 & & \\
\hline 1. & Трудоемкость изготовления & 19 & 20 & 19 & 17 & 19 & 94 & 18,8 \\
\hline 2. & $\begin{array}{l}\text { Себестоимость изделия } \\
\text { (технологическая) }\end{array}$ & 18 & 19 & 18 & 17 & 19 & 91 & 18,2 \\
\hline 3. & Собираемость конструкции & 6 & 9 & 8 & 7 & 6 & 36 & 7,2 \\
\hline 4. & Сложность сборки & 11 & 9 & 10 & 12 & 10 & 52 & 10,4 \\
\hline 5. & Унификация & 14 & 14 & 11 & 13 & 15 & 67 & 13,4 \\
\hline 6. & Стандартизация & 10 & 10 & 7 & 9 & 11 & 47 & 9,4 \\
\hline 7. & Повторяемость элементов & 12 & 9 & 10 & 10 & 11 & 52 & 10,4 \\
\hline 8. & Устанавливаемые размеры & 3 & 2 & 4 & 3 & 4 & 16 & 3,2 \\
\hline 9. & Освоенность технологии & 10 & 6 & 7 & 9 & 7 & 39 & 7,8 \\
\hline 10. & Повторяемость марок провода & 7 & 4 & 5 & 4 & 2 & 22 & 4,4 \\
\hline 11. & Регулируемость схемы & 1 & 3 & 4 & 2 & 2 & 12 & 2,4 \\
\hline 12. & Контролепригодность конструкции & 2 & 3 & 3 & 3 & 10 & 21 & 4,2 \\
\hline 13. & Масса изделия & 3 & 2 & 1 & 1 & 1 & 8 & 1,6 \\
\hline 14. & Удельная материалоемкость изделия & 13 & 13 & 9 & 9 & 7 & 51 & 10,2 \\
\hline 15. & Стоимость покупных элементов & 14 & 17 & 16 & 17 & 16 & 80 & 16 \\
\hline 16. & $\begin{array}{l}\text { Прогрессивные формообразующие } \\
\text { части деталей }\end{array}$ & 6 & 5 & 4 & 9 & 6 & 30 & 6 \\
\hline 17. & $\begin{array}{l}\text { Применяемость типовых } \\
\text { технологических процессов }\end{array}$ & 18 & 18 & 15 & 18 & 14 & 83 & 16,6 \\
\hline 18. & $\begin{array}{l}\text { Согласованность технологического } \\
\text { оснащения }\end{array}$ & 11 & 12 & 13 & 15 & 5 & 56 & 11,2 \\
\hline 19. & Применение групповых методов пайки & 5 & 11 & 12 & 11 & 13 & 52 & 10,4 \\
\hline
\end{tabular}

По каждому эксперту были вычислены коэффициенты Спирмэна и Диксона для повышения достоверности результатов и представлены в табл. 2.

Такие показатели, как трудоёмкость изготовления и технологическая себестоимость конструкции, являются комплексными. Они характеризуют важнейшие особенности конструкции разрабатываемого изделия и выбраны экспертами в первую очередь [9].

Таблица 2

\section{Результаты проверки статистической значимости выбора экспертов}

\begin{tabular}{|l|c|c|c|c|c|}
\hline \multirow{2}{*}{ Коэффициенты } & \multicolumn{5}{|c|}{ Эксперты } \\
\cline { 2 - 6 } & 1 & 2 & 3 & 4 & 5 \\
\hline Спирмэна, $\mathrm{p}_{\mathrm{k}}$ & 0,93 & 0,97 & 0,96 & 0,96 & 0,89 \\
\hline Диксона, $\mathrm{D}_{\mathrm{k}}$ & 0,47 & 1,0 & 0,92 & 0,94 & 0,0 \\
\hline $\begin{array}{l}\text { Результат проверки } \\
\text { условий, } \\
\mathrm{D}_{\mathrm{k}}>\mathrm{D}_{\text {нор }}(0,637)\end{array}$ & $\begin{array}{c}\text { Исключить } \\
\text { из выбора }\end{array}$ & $\begin{array}{c}\text { Использовать } \\
\text { в выборе }\end{array}$ & $\begin{array}{c}\text { Использовать } \\
\text { в выборе }\end{array}$ & $\begin{array}{c}\text { Использовать } \\
\text { в выборе }\end{array}$ & $\begin{array}{c}\text { Исключить } \\
\text { из выбора }\end{array}$ \\
\hline
\end{tabular}

В расчёт комплексного показателя технологичности конструкции были взяты показатели, указанные экспертами. А такие показатели, как трудоёмкость изготовления и технологическая себестоимость, использовались отдельно.

Для определения эффективности авторской методики показатели технологичности рассчитывались в соответствии с требованиями действующего стандарта ОСТ 4Г0.091.219 [9]. Согласно указанному нормативному документу, при расчёте значения интегрального показателя технологичности конструкции использовались показатели с жёстко заданными коэффициентами значимости. Расчётные значения показателей технологичности конструкции представлены в табл. 3. 
Разработка методологии оценки технологичности промышленных изделий...

601

ЭКОНОМИКА И ПРАВО

2021. Т. 31, вып. 4

Таблица 3

Сопоставительный анализ расчёта показателей технологичности конструкции изделия ЭЛИН-1 на этапе технического проекта по действующей отраслевой и разработанной методикам

\begin{tabular}{|c|c|c|c|c|c|c|}
\hline \multirow{2}{*}{$\begin{array}{l}\text { № } \\
\Pi / \Pi\end{array}$} & \multirow[b]{2}{*}{ Наименование показателя } & \multirow{2}{*}{$\begin{array}{c}\text { Значение } \\
\text { показателя }\end{array}$} & \multicolumn{2}{|c|}{$\begin{array}{c}\text { Значимость } \\
\text { согласно }\end{array}$} & \multicolumn{2}{|c|}{$\begin{array}{c}\text { Интегральный } \\
\text { показатель } \\
\text { технологич- } \\
\text { ности } \\
\text { согласно } \\
\end{array}$} \\
\hline & & & 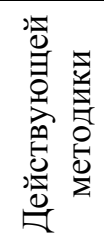 & 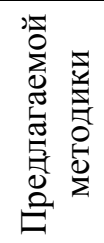 & 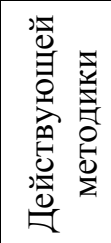 & 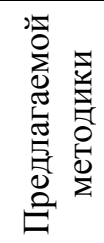 \\
\hline 1. & Использование микросхем & 0,123 & 0,257 & - & \multirow{13}{*}{0,611} & \multirow{13}{*}{0,577} \\
\hline 2. & Автоматизации монтажа & 0,861 & 0,255 & - & & \\
\hline 3. & Автоматизация подготовки элементов & 0,651 & 0,193 & - & & \\
\hline 4. & Автоматизация контроля и настройки & 0,754 & 0,132 & - & & \\
\hline 5. & Повторяемость элементов & 0,866 & 0,082 & - & & \\
\hline 6. & Применимость элементов & 0,825 & 0,047 & - & & \\
\hline 7. & Согласованность технологического оснащения & 0,784 & 0,031 & 0,146 & & \\
\hline 8. & Применение типовых техпроцессов & 0,567 & - & 0,188 & & \\
\hline 9. & Стоимость покупных элементов & 0,682 & - & 0,184 & & \\
\hline 10. & Унификация & 0,127 & - & 0,136 & & \\
\hline 11. & Применение групповых методов пайки & 0,483 & - & 0,124 & & \\
\hline 12. & Удельная материалоёмкость конструкции & 0,596 & - & 0,114 & & \\
\hline 13. & Сложность сборки & 0,790 & - & 0,113 & & \\
\hline
\end{tabular}

Как видно из табл. 3, показатели, выбранные экспертами, не предусмотрены для расчёта действующей методикой. А из отраслевых показателей выбран только один. Интегральный показатель технологичности оказался выше по значению. Предложенная авторская методика определения показателей технологичности позволила получить результат при оценке комплексного показателя изделия ЭЛИН-1 на этапе технического проекта.

Выбор системы показателей технологичности, подобранной для конкретного изделия в условиях опытно-серийного производства и с учётом организационно-технологических возможностей предприятия, позволяет более эффективно проводить оценку и отработку изделия на технологичность. В данном примере наибольшую значимость, по мнению экспертов, получили два показателя: коэффициент применения типовых техпроцессов и стоимость покупных ЭРЭ. Данные показатели выступили как индикаторы для принятия решения по конструированию изделия ЭЛИН-1. Конструкторами были предложены замены ряда ЭРЭ на более дешевые аналоги, от некоторых покупных элементов было предложено отказаться с внесением изменений в монтажную схему. В то же время стандартом ОСТ 4Г0.091.219 определён наиболее значимым показателем, а именно коэффициентом автоматизации и механизации монтажа. Эксперты не сочли данные показатели значимыми, так как монтаж печатных плат на предприятии реализован автоматизированным способом, поэтому повышения комплексного показателя ТК изделия ЭЛИН-1 рассмотрение данного показателей не дало бы.

Таким образом, предложенная авторская методика выбора показателей технологичности позволила получить наиболее достоверный результат на этапе технического проекта за счёт тщательного отбора показателей, учёта особенностей конструкции и технологического уровня предприятия, а также дала возможность выявить резервы повышения технологичности изделия. Применение данной методики способствует сокращению сроков и затрат, связанных с освоением и производством нового изделия в условиях опытно-серийного производства. 


\section{СПИСОК ЛИТЕРАТУРЫ}

1. ЕСТПП: справоч. пособие. М.: Изд-во стандартов, 1984.

2. ГОСТ 14.202-73. ЕСТПП. Правила выбора показателей технологичности конструкции изделий. М.: Изд-во стандартов, 1973.

3. Технологичность конструкции изделия: справочник / под ред. Ю.Д. Амирова. М.: Машиностроение, 1990.

4. Кононенко В.Г., Кушнаренко С.Г., Прялин М.А. Оценка технологичности и унификации машин. М.: Машиностроение, 1986.

5. Маликова Д.М. Роль машиностроения в конкурентоспособности страны // Сб. тез. докл. конгресса молодых ученых. Электронное издание. СПб.: Университет ИТМО, 2017.

6. Маликов В.Н. Отработка конструкции РЭА на технологичность: учеб. пособие. Харьков: Изд-во ХАИ, 1996.

7. Маликова Д.М. Современные подходы к управлению опытно-серийным производством на предприятиях оборонно-промышленного комплекса // Вестн. ИжГТУ. 2017. Т. 20, № 2. С. 118-121.

8. Курбанов М.М. Формирование системы показателей технологичности радиоэлектронных средств методом построения дерева декомпозиции. М.: МГУПИ, 2006.

9. ОСТ 4.091.171-81. ОСТПП. Номенклатура базовых показателей технологичности для бытовой РЭА и метод их расчета. М.: Изд-во стандартов, 1981.

Поступила в редакцию 11.06.2021

Маликова Диляра Мансуровна, кандидат экономических наук, доцент,

доцент кафедры «Экономика и управление организацией»

ФГБОУ ВО «Ижевский государственный технический университет им. М.Т. Калашникова»

426069, Россия, г. Ижевск, ул. Студенческая, д. 7

E-mail: info@istu.ru

\section{D.M. Malikova \\ DEVELOPMENT OF A METHODOLOGY FOR ASSESSING THE MANUFACTURABILITY OF INDUSTRIAL PRODUCTS IN THE CONDITIONS OF PILOT PRODUCTION}

DOI: $10.35634 / 2412-9593-2021-31-4-597-602$

The article is devoted to improving the reliability of a comprehensive quantitative assessment of the technological performance of the product design in the conditions of pilot production, taking into account the specific production and technological conditions of the enterprise by solving the problem of objective selection of technological performance indicators. To achieve this goal, the following tasks were solved: the indicators of the manufacturability of product designs, their quantitative assessment were studied; a mathematical model for the formation of design indicators of manufacturability was developed, the optimal set of indicators of manufacturability for a particular product was selected; a method for quantitative evaluation and expert selection of indicators of manufacturability by their weights was developed; an algorithm for determining the integral indicator of the product's manufacturability was developed; the developed algorithm for the integral evaluation of the product's manufacturability indicators was applied. The practical significance of the work lies in the approbation of the author's method of expert selection and the determination of the integral index of manufacturability. This indicator is one of the elements of improving the efficiency of the enterprise, especially in the conditions of pilot production. The method developed by the author makes it possible to more reliably assess the manufacturability of the product at the initial stages of design. A positive result was obtained by expert selection of indicators that take into account the design features and the technological level of the manufacturer. In addition, the proposed method made it possible to identify reserves for improving the manufacturability of the product design. The use of the author's methodology helps to reduce the time and costs associated with the development and production of a new product.

Keywords: pilot production, manufacturability of a product, expert assessment, ranking of indicators, increasing manufacturability, economic efficiency, financial result, labor process.

Malikova D.M., Candidate of Economics, Associate Professor,

Associate Professor at Department of Economics and Company Management

Kalashnikov Izhevsk State Technical University

Studencheskaya st., 7, Izhevsk, Russia, 426069

E-mail: info@istu.ru 